\title{
Performance Improvement in Gradient based Algorithm for the Estimation of Fingerprint Orientation Fields
}

\author{
Meghna B. Patel \\ Assistant Professor, AMPICS \\ Ganpat University, \\ Kherva, India
}

\author{
Satyen M. Parikh, PhD \\ Dean, FCA \\ Ganpat University, \\ Kherva, India
}

\author{
Ashok R. Patel, PhD \\ Keiser University, \\ Lakeland, 33805, \\ Florida, USA
}

\begin{abstract}
Accuracy of fingerprint recognition system is reliable for correct measurement of fingerprint features. Orientation estimation of fingerprint ridges is playing a vital role in image enhancement, segmentation, classification and recognition. The accurate estimation of ridge orientation improves the performance of minutiae extraction and matching algorithm. The noisy fingerprint does not contain the clear ridge structure, that's why ridge orientation estimation is the toughest and challenging task in fingerprint image enhancement. Gradientbased orientation estimation algorithm is widely adopted and most popular method accepted in literature. This paper enhance the consistency level of ridge orientation after changing the range of output direction from $[-\mathrm{PI} / 4, \mathrm{PI} / 4]$ to $[0, \mathrm{PI}]$ and remove the inconsistency. The implementation is done using java language and the experimental result is made on FVC2000 and FingerDOS databases. The outcome of enhanced new method for estimating ridge orientation give better performance than the existing gradient based approach.
\end{abstract}

\section{Keywords}

Fingerprint recognition, fingerprint enhancement, orientation estimation

\section{INTRODUCTION}

The frequently increasing requirements of security, biometric technologies have been used widely. For personal identification most reliable and popular technique is the fingerprint recognition among all biometric techniques [1] because the pattern of fingertip is remain unique even for twins, require less storage space and low cost fingerprint sensor. Fingerprint recognition system is used from simple

access control to convicting criminal by forensic and law agencies [2][3]. Lots of research is done on fingerprint recognition and many researchers proposed no. of proven methods. Though, recent literatures demonstrate that still require developing new robust system. Even the main important research issue is the poor quality images give the low performance [3]. Because in reality the quality of the fingerprint is weak due to scare and cuts, dry skin, sensor noise, give high or low pressure on sensor create a noise, and weak ridge and valley pattern of fingerprint, partial fingerprints and latent fingerprints contain complex background and so on.

Image enhancement process improves the quality of an image and ultimately improves the performance of fingerprints recognition systems. The fingerprints made up of two types of features like: minutia or singular points consider as local features and a ridge pattern orientation and frequency consider as global features.
To verify or identify an individual local features are used while the global features describe local orientation of ridges and valleys using orientation fields. Ridge orientation is an important feature because the impact of it is affected to other processes like fingerprint enhancement, classification, segmentation and matching. The ridge orientation can be used in [4-9] image enhancement algorithms, singular points detection [5][10-12][16] and classification [13-19]. If accurate orientation estimation is not found then false recognition is generate.

\section{RELATED WORK ON ORIENTATION ESTIMATION}

In an image of fingerprint, orientation field indicate the direction of ridges. During literature survey found many methods for ridge orientation estimation. There are many methods are found on gray-scale relationship between pixels [3][21-29,41]. There are many different way to compute the ridge orientation like [22] calculated gray consistency beside 16 directions at every pixel and the orientation of the best consistency is taken as the ridge orientation. Some of them separated pixels into ridge and non-ridge pixels and determined ridge orientation by calculating the consistency of pixel type [23]. Also using the neural network evaluated the exactness of ridge orientation [25][33]. Among all this famous method for ridge orientation estimation is the gradient-based method [3][29-32][39-40].

The acquired values are perfect compared to pixel-alignment method [1][9] that is the main benefit of this algorithm. The ridge orientation estimation is totally depended on relationship of gradient among neighbor pixels. However, the gradients are the orientations at the pixel scale, the orientation of the ridge is orthogonal to the average phase angle of pixels value changes, indicated through gradients. However, only ridge edge gradients are orthogonal to the ridge. Therefore the gradients averaging step is necessary.

\section{ORIENTATION ESTIMATION}

The implementation of gradient-based method for orientation estimation is done using following steps [35].

- The fingerprint image should be dividing into $\mathrm{WxW}$ size blocks.

- In every block for each pixel calculate the gradients $\mathrm{Gx}$ and Gy.

- Use the below equations (1) and (2) for determine the local orientation at each pixel $(i, j)$. 


$$
\begin{aligned}
& V x(i, j)=\sum_{u=i-\frac{w}{2}}^{i+\frac{w}{2}} \sum_{v=j-\frac{w}{2}}^{j+\frac{w}{2}} 2 G x(u, v) G y(u, v), \\
& V y(i, j)=\sum_{u=i-\frac{w}{2}}^{i+\frac{w}{2}} \sum_{v=j-\frac{w}{2}}^{j+\frac{w}{2}}\left(G^{2} x(u, v)-G^{2} y(u, v)\right), \\
& \theta(i, j)=\frac{1}{2} \tan ^{-1}\left(\frac{V x(i, j)}{V y(i, j)}\right)
\end{aligned}
$$

Where, the size of the local window is denoted as $\mathrm{W}$, and the gradient magnitudes in $\mathrm{x}$ and $\mathrm{y}$ directions are denoted as $\mathrm{Gx}$ and Gy.

- Using the following equation (3), the consistency level of orientation field is estimated for the local neighborhood of a block(i, j).

$$
C(i, j)=\frac{1}{N} \sqrt{\sum_{\left(i^{\prime}, j^{\prime}\right) \in D}\left|\theta\left(i^{\prime}, j^{\prime}\right)-\theta(i, j)\right|^{2}},
$$

$$
\left|\theta^{\prime}-\theta\right|=\left\{\begin{array}{l}
\left.d \text { if } \mathrm{d}=\left(\theta^{\prime}-\theta+360\right) \bmod 360\right)<180 \\
\quad \text { otherwise }
\end{array}\right.
$$

Where, D represents the local neighborhood around the block (i,j) (the size of D is taken $5 \mathrm{X} 5 ; \mathrm{N}$ is the number of blocks within $\mathrm{D} ; \boldsymbol{\theta}^{\prime}\left(i^{\prime}, j^{\prime}\right)$ and $\boldsymbol{\theta}(\boldsymbol{i}, j)$ are local ridge orientation at blocks $\left(i^{\prime}, j^{\prime}\right)$ and $(i, j)$ respectively.

- If the consistency level is above a certain threshold Tc, then the local orientations around this region are reestimated at a lower resolution level until $\mathrm{C}(\mathrm{i}, \mathrm{j})$ is below a certain level.

The equation (3) is implemented for calculate the consistency of the orientation field. But the last point "If the consistency level is above a certain threshold Tc, then the local orientations around this region are re-estimated at a lower resolution level until $\mathrm{C}(\mathrm{i}, \mathrm{j})$ is below a certain level". The author did not mentioned about clear threshold value and did not mention about that if can't find a certain value, what is the standard to decrease the neighborhood block $\mathrm{D}$ until find a certain level of $\mathrm{C}(\mathrm{i}, \mathrm{j})$. It is difficult to implement the last step in this algorithm. That's why this step is neglecting and apply only equation (2). But because of omitting equation (3) inconsistency is in an orientation.

Then after found that the output direction range is [-PI/4, PI/4] and this range makes the inconsistency in an orientation because the angle between $-\mathrm{PI} / 4$ to PI/4 can't contain all the direction in a fingerprint image. After research found the below equation (4) which converts the angle [36]. Using it convert the range $[-\mathrm{PI} / 4, \mathrm{PI} / 4]$ to $[0$ to $\mathrm{PI}]$ and remove the inconsistency.

$$
\theta=\frac{1}{2} \pi+\frac{1}{2}\left\{\begin{array}{l}
\arctan \left(\frac{G_{B y}}{G_{B x}}\right), G_{B x} \geq 0, \\
\arctan \left(\frac{G_{B y}}{G_{B x}}\right)+\pi, G_{B x}<0 \cap G_{B y} \geq 0, \\
\arctan ^{-1}\left(\frac{G_{B y}}{G_{B x}}\right)-\pi, G_{B x}<0 \cap G_{B y}<0
\end{array}\right.
$$

\section{EXPERIMENTAL RESULTS AND ANALYSIS}

The Implementation of an algorithm is done using java language and for the experiments the databases FVC 2000 DB1, DB2, DB3, DB4 released on the web [37], and FingerDOS database [38] are used. The details of the databases are given in below Table 1 and Table 2. For showing the result 10 fingerprints are taken from the each cluster of the databases. The performance of the proposed method should be checked by two criteria.

Criteria-I: - Removal of Inconsistency

Criteria-II:- Execution Time

\begin{tabular}{|c|c|c|c|c|}
\hline \multicolumn{5}{|c|}{ FVC2000 } \\
\hline Set B & $\begin{array}{l}\text { Sensor } \\
\text { Type }\end{array}$ & $\begin{array}{l}\text { Image } \\
\text { Size }\end{array}$ & $\begin{array}{c}\text { No. of } \\
\text { Impression }\end{array}$ & Resolution \\
\hline DB1 & $\begin{array}{l}\text { Low-cost } \\
\text { Optical } \\
\text { Sensor }\end{array}$ & $300 \times 300$ & $10 x 8$ & 500 dpi \\
\hline DB2 & $\begin{array}{c}\text { Low-cost } \\
\text { Capacitive } \\
\text { Sensor }\end{array}$ & $256 \times 364$ & $10 \times 8$ & 500 dpi \\
\hline DB3 & $\begin{array}{l}\text { Optical } \\
\text { Sensor }\end{array}$ & $448 \times 478$ & $10 x 8$ & 500 dpi \\
\hline DB4 & $\begin{array}{l}\text { Synthetic } \\
\text { Generator }\end{array}$ & $240 \times 320$ & $10 x 8$ & $\begin{array}{c}\text { about } 500 \\
\text { dpi }\end{array}$ \\
\hline
\end{tabular}

The below Table 3 shows the result, that the inconsistency should be remove after changing the range of output direction

\begin{tabular}{|c|c|c|c|}
\hline \multicolumn{4}{|c|}{ FingerDOS } \\
\hline FingerDOS & $\begin{array}{c}\text { Image } \\
\text { Size }\end{array}$ & $\begin{array}{c}\text { No. of } \\
\text { Impression }\end{array}$ & Resolution \\
\hline $\begin{array}{c}\begin{array}{c}\text { optical } \\
\text { sensor }\end{array} \\
\text { (SecuGeniD- } \\
\text { USB SC) }\end{array}$ & $260 \times 300$ & $\begin{array}{l}3600=60 \times 6 \times 10 \\
\text { i.e. } \\
\text { No. of } \\
\text { subjects }=60 \\
\text { No. of fingers=6 } \\
\text { (index, middle } \\
\text { and thumb of } \\
\text { right and left } \\
\text { hand) } \\
\text { No. of } \\
\text { impression }=10\end{array}$ & 500 PPI \\
\hline
\end{tabular}
from $[\mathrm{PI} / 4, \mathrm{PI} / 4]$ to $[0, \mathrm{PI}]$. And the Table 4 shows the summary of the execution time that prove that the proposed method give the better result.

Table 1. FVC2000 Database][37]

Table 2. FingerDOS Database[38] 
Table 3. Result Analysis on FVC2000 Database and FingerDos Database

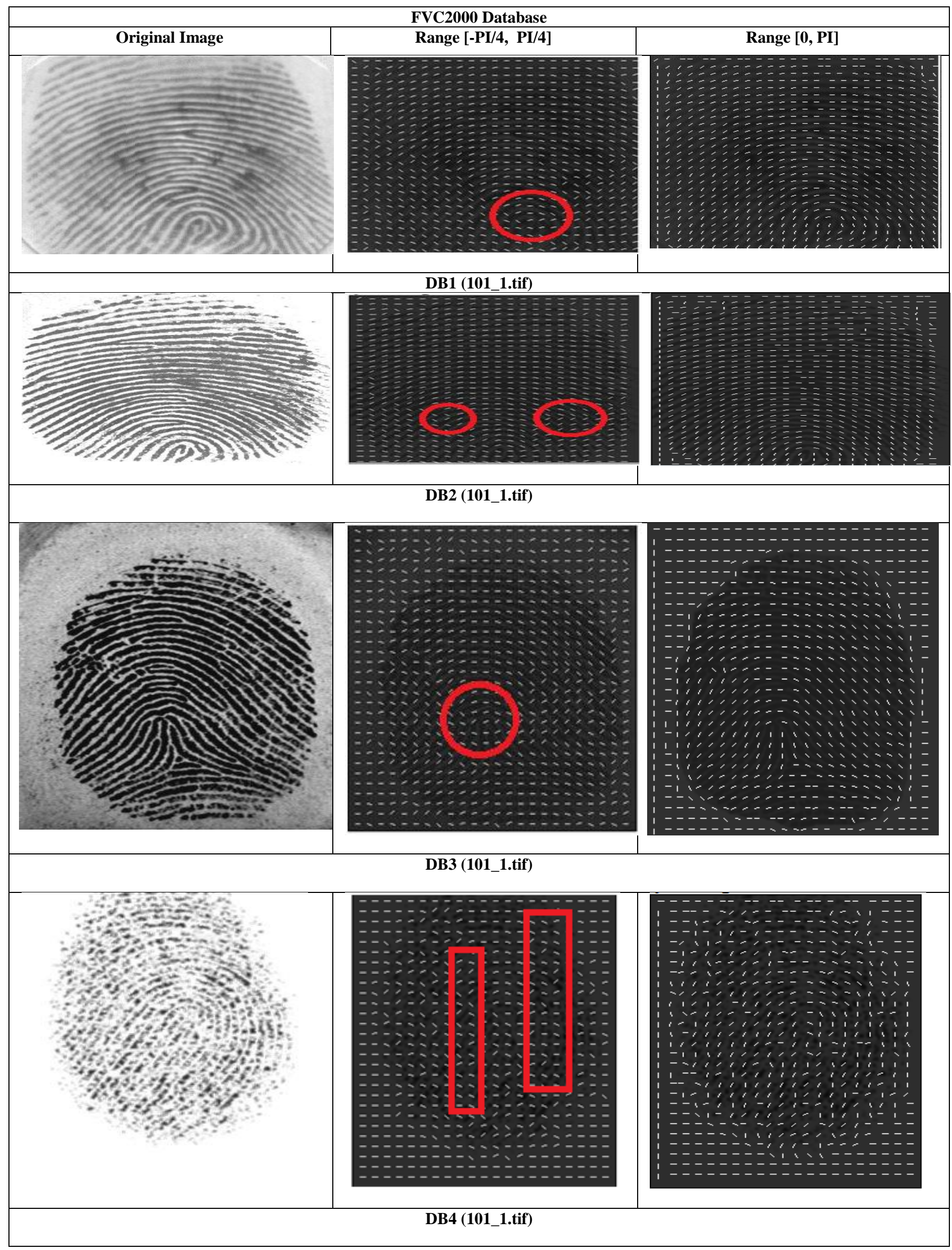




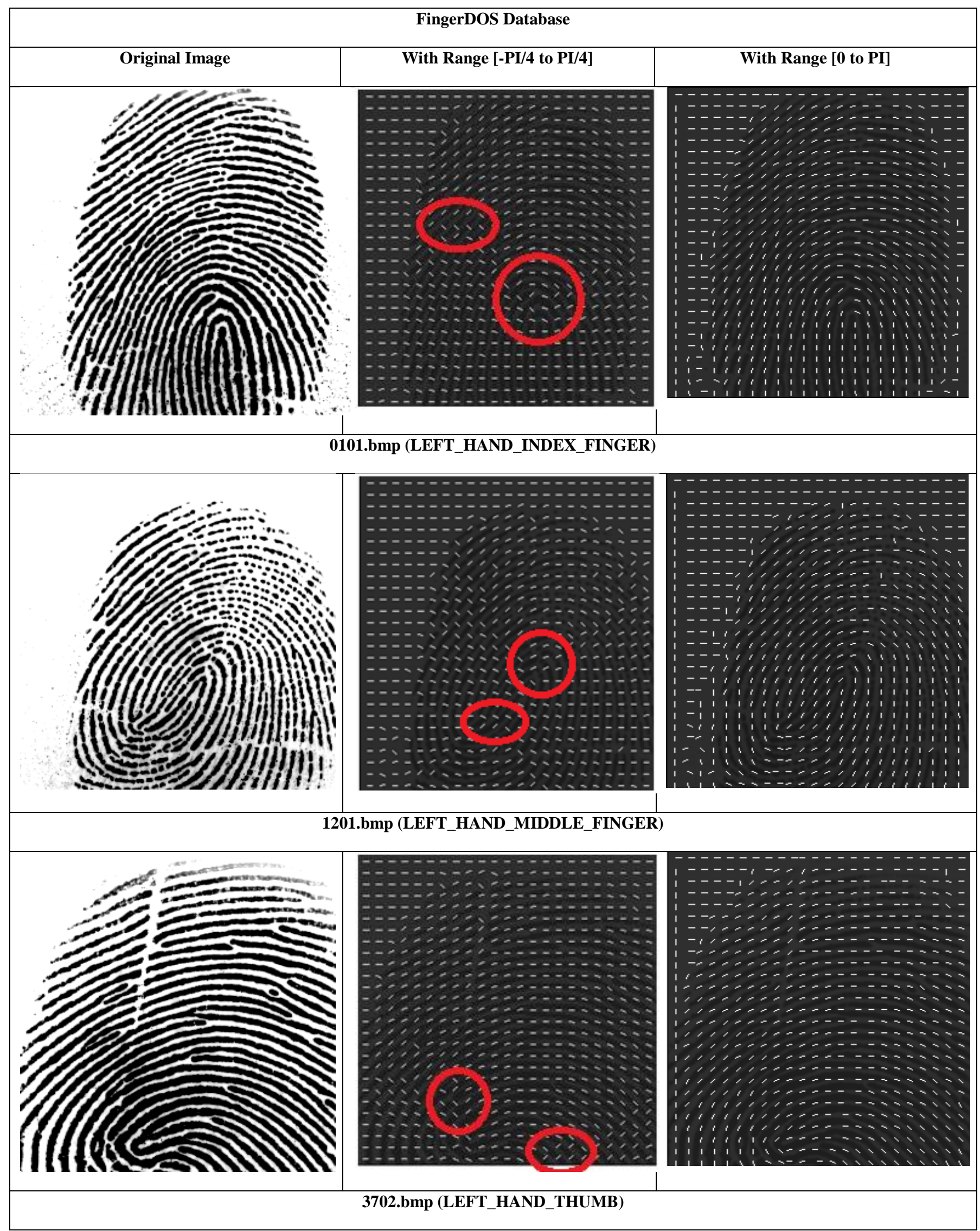



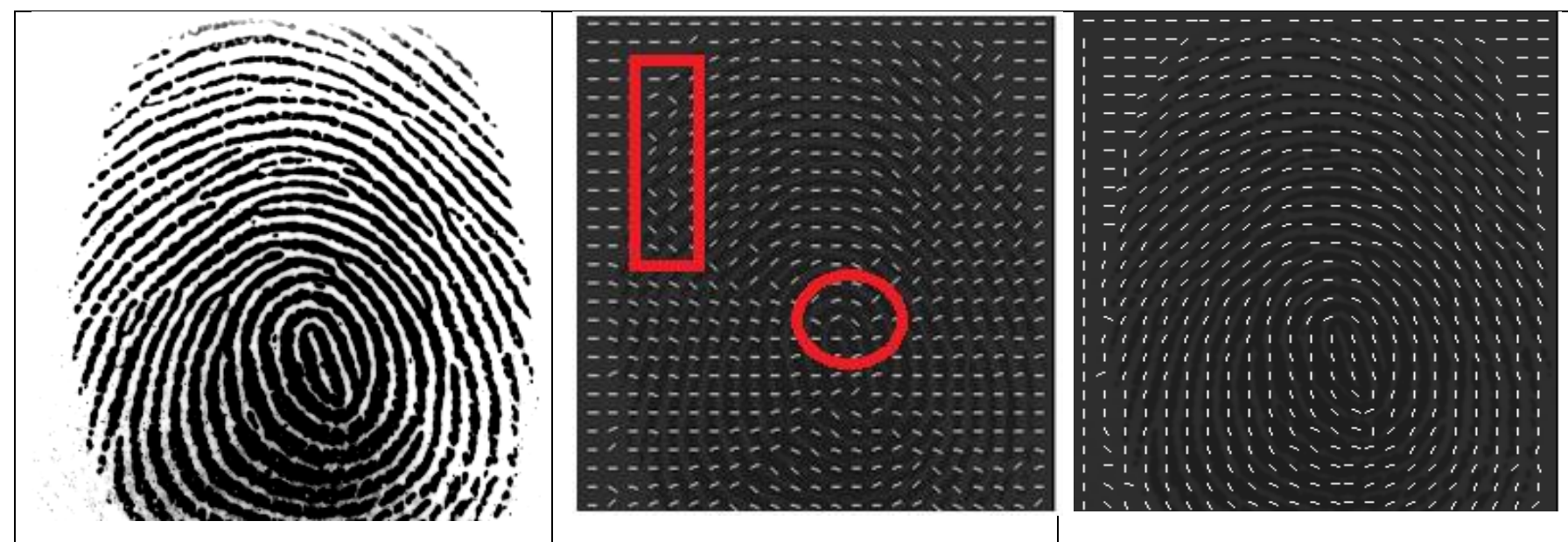

0401.bmp (RIGHT_HAND_INDEX_FINGER)
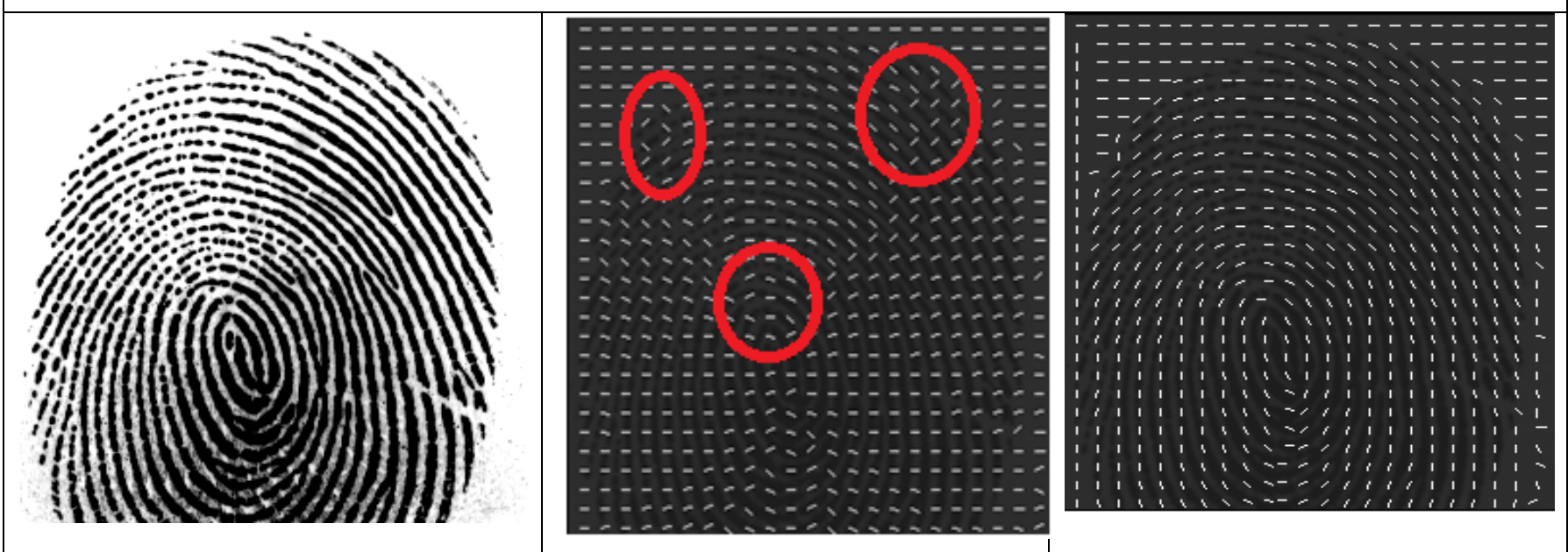

1201.bmp (RIGHT_HAND_MIDDLE_FINGER)
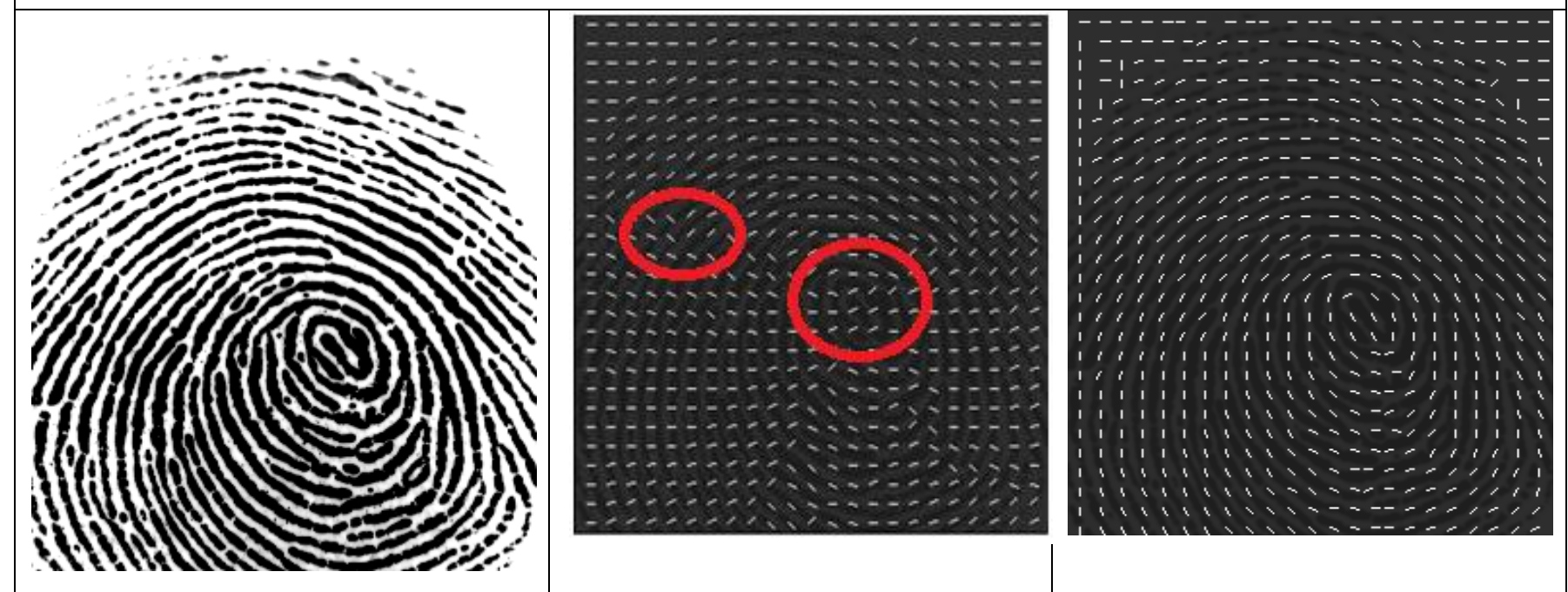

3901.bmp (RIGHT_HAND_THUMB) 
Table 4. Summary of an Execution Time (in milliseconds)

\begin{tabular}{|l|c|c|}
\hline \multicolumn{1}{|c|}{$\begin{array}{c}\text { Images of FVC2000 and FingerDos } \\
\text { Databases }\end{array}$} & $\begin{array}{c}\text { Range } \\
\text { [-PI/4, PI/4] }\end{array}$ & $\begin{array}{c}\text { Range } \\
{[\mathbf{0 ,} \text { PI] }}\end{array}$ \\
\hline FVC2000_DB1_B_101_1.tif & 31 & 15 \\
\hline FVC2000_DB2_B_101_1.tif & 62 & 47 \\
\hline FVC2000_DB3_B_101_1.tif & 156 & 78 \\
\hline FVC2000_DB4_B_101_1.tif & 78 & 62 \\
\hline LEFT_HAND_INDEX_FINGER_0101.bmp & 109 & 62 \\
\hline LEFT_HAND_MIDDLE_FINGER_1201.bmp & 63 & 15 \\
\hline LEFT_HAND_THUMB_3702.bmp & 47 & 32 \\
\hline RIGHT HAND_INDEX_FINGER_0401.bmp & 31 & 31 \\
\hline RIGHT_HAND_MIDDLE_FINGER_1201.bmp & 62 & 15 \\
\hline RIGHT_HAND_THUMB_3901.bmp & 62 & 47 \\
\hline
\end{tabular}

\section{CONCLUSION AND FUTURE WORK}

Fingerprint ridge orientation is playing vital role in many processes like fingerprint image enhancement, classification of an image, core and delta point detection. That's why necessary to estimate correct orientation. Fingerprint image contains many noisy regions or blocks with singular points which hardly generate the correct orientation, because of this reason gradient based orientation estimation algorithm can't guarantee for correctness of ridge orientation. And the incorrect orientation gives false singular points, classification and ridge flows. This paper enhances the performance of gradient based orientation estimation algorithm and proposes new and reliable fingerprint orientation estimation without inconsistency. In future work try to implement and improve the performance enhancement algorithms.

\section{REFERENCES}

[1] Biometrics Market And Industry Report 2009-2014. International Biometric Group, New York, 2009.

[2] Cappelli R., Maio D., Wayman J. L., Jain A. K., Performance Evaluation Of Fingerprint Verification Systems. IEEE Transactions On Pattern Analysis And Machine Intelligence, 2006, Vol. 28, No. 1, Pp. 3-18.

[3] Hong L., Jain A. K., Wan Y., Fingerprint Image Enhancement: Algorithm And Performance Evaluation. IEEE Trans. On Pattern Analysis And Machine Intelligence, 1998, Vol. 20, No. 8, Pp. 777-789.

[4] Chikkerur S., Cartwright A. N., Govindaraju V., Fingerprint Enhancement Using Stft Analysis. Pattern Recogn. 2007, Vol. 40, No. 1, Pp. 198-211.

[5] Yi Wang, Jiankun Hu, Damien Phillips, “A Fingerprint Orientation Model Based On 2d Fourier Expansion (Fomfe) And Its Application To Singular-Point Detection And Fingerprint Indexing", IEEE Transactions On Pattern Analysis And Machine Intelligence, Vol. 29, No. 4, Pp.573-585, 2007.

[6] CarstenGottschlich, PredaMihailesc, Axel Munk, "Robust Orientation Field Estimation And Extrapolation Using Semilocal Line Sensors", IEEE Transactions On Image And Signal Processing And Analysis, Pp. 529-533. 2009.
[7] S. Yoon, J. Feng, A. Jain, J.2011. Latent Fingerprint Enhancement Via Robust Orientation Field Estimation. In Proceedings Of IJCB( International Joint Conference On Biometrics.

[8] Jianjiang Feng, Jie Zhou, Anil K. Jain, "Orientation Field Estimation For Latent Fingerprint Enhancement", IEEE Transactions On Pattern Analysis And Machine Intelligence, Vol. 54, No. 4, Pp. 925-940, 2013.

[9] Prasad Reddy P.V.G.D, M. James Stephen, "Towards Accurate Estimation Of Fingerprint Ridge Orientation Using Bpnn And Ternarization", Iosr Journal Of Computer Pp.2278-8727, Vol 13,. 2013.

[10] Liu M., Jiang X., Kot A. C., Fingerprint Reference-Point Detection. Eurasip J. Appl. Signal Process. 2005, Pp. 498 509.

[11] Bazen A. M., Gerez S. H. Systematic Methods For The Computation Of The Directional Fields And Singular Points Of Fingerprints. IEEE Trans. Pattern Anal. Mach. Intell., 2002, Vol. 24, No. 7, Pp. 905-919.

[12] Wrobel K., Doroz R., New Method For Finding A Reference Point In Fingerprint Images With The Use Of The IPAN99 Algorithm. Journal Of Medical Informatics \& Technologies. Vol. 13, Pp. 59-64, 2009.

[13] Costa S. M., Fernandez F. J., Oliveira J. M., A New Paradigm On Fingerprint Classification Using Directional Image. Sibgrapi, 405, 2002.

[14] Halici U., Ongun G., Fingerprint Classification Through Self-Organizing Feature Maps Modified To Treat Uncertainties. Proc. Of The IEEE, 1996, Vol. 84, No. 10 , Pp. 1497-1512.

[15] Hong L., Jain A. K., Prabhakar S., A Multichannel Approach To Fingerprint Classification. IEEE Trans. Pattern Anal.Mach.Intell. 1999, Vol. 21, No. 4, Pp. 348359.

[16] Jain A. K., Karu K., Fingerprint Classification. Pattern Recognition, 1996, Vol. 29, No. 3, Pp. 38-44. 
[17] Davide Maltoni, Dario Maio, Anil K. Jain, Salil Prabhakar, L. L. 2009. Handbook Of Fingerprint Recognition.

[18] S. Dass, "Markov Random Field Models For Directional Field And Singularity Extraction In Fingerprint Images", IEEE Trans. Image Processing, Vol. 13, No. 10, Pp. 1358-1367, 2004.

[19] Jie Zhou, JinweiGu, "A Model-Based Method For The Computation Of Fingerprints' Orientation Field", IEEE Transactions On Image Processing, Vol. 13, No. 6, June 2004.

[20] Qinzhi Zhang, Hong Yan, "Fingerprint Orientation Field Interpolation Based On The Constrained Delaunay Triangulation", International Journal Of Information And Systems Sciences Volume 3, Number 3, Pages 438-452, 2007.

[21] Kawagoe M., Tojo A., Fingerprint Pattern Classification, Pattern Recognition, 1984, 17(3): 295-303.

[22] Mehtre B.M., Murthy N.N., Kapoor S., Segmentation Of Fingerprint Images Using The Directional Image, Pattern Recognition, 1987, 20(4): 429-435.

[23] Hung D.C.D., Enhancement And Feature Purification Of Fingerprint Images, Pattern Recognition, 1993, 26(11): 1661-1672.

[24] Kovacs-Vajnazs.M.,Rovatti R., Frazzoni M., Fingerprint Ridge Distance Computation Methodologies, Pattern Recognition, 2000, 33: 69-80.

[25] Nagaty K.A, On Learning To Estimate The Block Directional Image Of A Fingerprint Using A Hierarchical Neural Network, Neural Networks, 2003, 16:133-144.

[26] Rao, K., Black, K., Type Classification Of Fingerprints: A Syntactic Approach, IEEE Transaction On Pattern Analysis And Machine Intelligence, 1980, 2(3):223-231.

[27] Halici, L., Ongun, G., Fingerprint Classification Through Selforganizing Feature Maps Modified To Treat Uncertainties, Proceedings Of The IEEE , 1996, 84(10): $1497-1512$.

[28] Donahue M.J., Rokhlin S.I., On The Use Of Level Curves In Image Analysis, Image Understanding, 1993, 57(2): 185-203.

[29] Ratha N., Chen S., Jain A.K., Adaptive Flow OrientationBased Feature Extraction In Fingerprint Images, Pattern Recognition, 1995, 28(11):1657-1672

[30] Bazen A.M., Gerez S.H., Systematic Methods For The Computation Of The Directional Fields And Singular
Points Of Fingerprints, IEEE Transactions On Pattern Analysis And Machine Intelligence, 2002, 24(7): 905-919.

[31] Maio D., Maltoni D., Direct Gray-Scale Minutiae Detection In Fingerprints, IEEE Transactions On Pattern Analysis And Machine Intelligence, 1997, 19(1): 27-39.

[32] Almansa A., Lindederg T., Fingerprint Enhancement By Shape Adaptation Of Scale-Space Operators With Automatic Scale Selection, IEEE Transaction On Image Processing, 2000,9(12):2027-2042

[33] E. Zhu, J. Yin, C. Hu, And G. Zhang. A Systematic Method For Fingerprint Ridge Orientation Estimation And Image Segmentation. Pattern Recognition, 39(8):1452 $1472,2006$.

[34] Kass M., Witkin A., Analyzing Orientated Pattern. Computer Vision, Graphics And Image Processing, 1987, Vol. 37, Pp. 362-397.

[35] Anil Jain AndSharathPankanti. Fingerprint Classification And Matching[Online] Available: Http://Citeseerx.Ist.Psu.Edu/Viewdoc/Download?Doi=10. 1.1.83.3331\&Rep=Rep1\&Type $=$ Pdf

[36] Yuan Mei, Huaijiang Sun, AndDeshen Xia (2006). A Gradient-Based Robust Method For Estimation Of Fingerprint Orientation Field, Image And Vision Computing, Volume 27, Issue 8, 2 July 2009, Pages 1169-1177

[37] D. Maio, D. Maltoni, R. Capelli, J. L. WaymanAnd A. K. Jain,"Fvc2000: Fingerprint Verification Competition", IEEE Trans. PatternAnal. Mach. Intell., Vol. 24, No. 3, Pp. 402-412, 2002.

[38] F. Francis-LothaiAnd D. B. L. Bong, "Fingerdos: A Fingerprint Database Based On Optical Sensor," Wseas Transactions On Information Science And Applications, Vol.12, No. 29, Pp. 297-304, 2015

[39] J. Yang, L. Liu, T. Jiang, and Y. Fan, "A modified Gabor filter design method forfingerprint image enhancement," Pattern Recognition Letter, Vol. 24, 2003, pp. 1805-1817.

[40] J. Cheng and J. Tian, "Fingerprint enhancement with dyadic scale-space," PatternRecognition Letters, Vol. 25 , 2004, pp. 1273-1284.

[41] Ms. Meghna B. Patel , Dr. Satyen M. Parikh , Dr. Ashok R. Patel, "Performance Improvement in Binarization for Fingerprint Recognition", IOSR Journal of Computer Engineering (IOSR-JCE) e-ISSN: 2278-0661,p-ISSN 2278-8727, Volume 19, Issue 3, Ver. II (May.-June. 2017), PP 68-74 www.iosrjournals.org 